\title{
The Optimal Search Region in the Sea
}

\section{Xiaolei Zhang}

School of North China Electric Power University, Baoding 071000, China;

837761034@qq.com

Keywords: Multi-objective optimization, Normal distribution, maritime search and rescue

\begin{abstract}
The major assumption is that the main body of the crashed plane is complete and don't drift with flow. All the work units are not affected by weather conditions and obey the unified dispatch.

The model, Determining search region, is based on the theory that the crashed plane obeys normal distribution on both sides of the baseline which we determine according to the predetermined route and other information about the crashed plane. After the baseline determined, we use the work units' affordability, the probability density of the target's distribution, the probable error and the crashed plane's distance of unpowered gliders as constraints to find the optimal search width.
\end{abstract}

\section{Introduction}

In recent years the frequency of aircraft accidents has risen rapidly such as MH370. Due to the lack of relevant search experience and in the absence of good cooperation between the countries, finally the progress is a very slow search and the search result is far from meeting expectations. How to search and rescue better at sea with limited time and resource is a problem worth considering.

Our mathematical model is based on the establishment of the above-described problems. Within a defined area, we developed an optimal search plan .The point is that by understanding the relevant search criteria and specific scenarios we set one of the best search region.

\section{The Model to Determine Search Region}

Terminology and Definition.

Table 1. Terminology and definitions

\begin{tabular}{cc}
\hline$A$ & Search region \\
\hline$A^{\prime}$ & Alternative search region \\
$L$ & The best search width[1] \\
$l$ & Reference baseline [2] \\
$V_{i}$ & The length of reference baseline \\
$T_{i}$ & The flying speed of search aircraft \\
$W_{i}$ & Work unit's maximum flight time \\
$Z_{i}$ & Work unit's detection width \\
$y_{1}$ & Work unit's detection ability[3] \\
$y_{2}$ & The crashed plane's last contact point \\
$r_{i}$ & The crashed plane's maximum range \\
$\varepsilon$ & along the route \\
$\sigma$ & The affordable search width of work unit \\
$E$ & Probable error \\
& The total probable error of position
\end{tabular}


$X$
$Y$
$C_{y}$
$\rho$
$S$
$E^{\prime}$
Initial target position error

Search facility position error

Crashed plane's lift coefficient

Air density

Crashed plane wing area

The distance of crashed plane unpowered glider

Establish a Baseline. Regard the point where the console contract with the crashed plane last time as a starting point $y_{1}$.End of the maximum range is the end point $y_{2}$.Set the aircraft's predetermined routes as a baseline $I$ and its length is $L$.

Determine the Best Search width R. Ignoring the effect of weather conditions, aircraft search capabilities can be deployed from a search speed $V$, search duration $T$, and scan width $W$ to determine. The maximum width each search aircraft can withstand:

$$
r_{i}=\frac{W_{i} L}{V_{i} T_{i}}
$$

The maximum width search teams can withstand:

$$
r=\sum r_{i}
$$

In the case that some of the government provide resources. The maximum width search teams can withstand become $k r, k$ is determined by the maximum resources government can provide. So the first constraint is:

$$
R \leq k r
$$

Search target is evenly distributed along the reference baseline and it obeys normal distribution along the direction perpendicular to the reference baseline. Generally speaking, left and right sides of the reference baseline is the same probability distribution. Baseline $l$ is used as the axis $y$ of a Coordinate system. Start and end points are respectively $\left(0, y_{1}\right)$ and $\left(0, y_{2}\right)$.Make $(x, y)$ represents the target true position. Then when the $x$ and $y$ are independent, the $x$ obeys normal distribution and the symmetry axis is $I$, just as the Figure 2 shows. The probability density function is:

$$
\begin{gathered}
f(x)=\frac{1}{2 \pi \sigma^{2}} \exp \left(-\frac{x^{2}}{2 \sigma^{2}}\right), \quad-\infty<x<+\infty \\
f(y)= \begin{cases}\frac{1}{y_{2}-y_{1}}, y_{1}<y<y_{2} \\
0, \quad \text { others }\end{cases}
\end{gathered}
$$
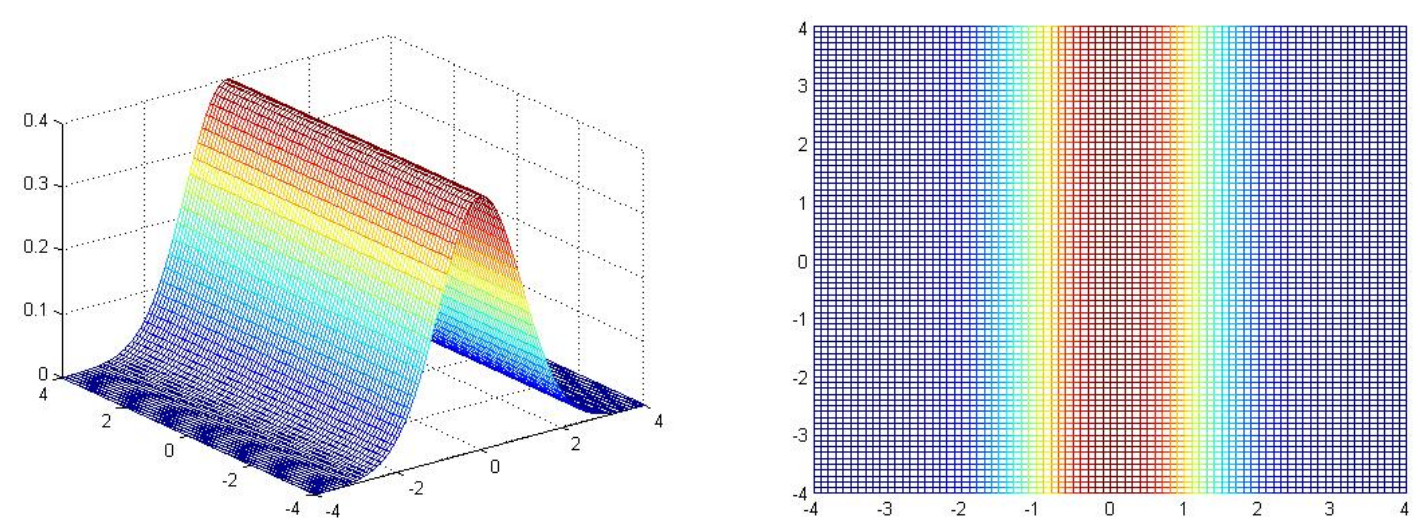

Figure 1.The probability density distribution of the search target 
In the direction of the shaft $x$, the longer from the baseline $I$ (i.e. Axis $y$ ), the smaller the target' s probability of containing is. The target's probability of containing on the both sides of baseline is expressed as:

$$
P O C=1-\exp \left(-\frac{x_{r}^{2}}{2}\right)
$$

$x_{r}$ is the vertical distance from baseline.

The standard variance $\sigma$ is used to measure the target position offsetting reference points of the size of the error. But in the maritime search and rescue, we commonly use probable error $\varepsilon$ to measure the size of the target position error. $\sigma$ usually makes $x_{r}$ whose constraint is that $P O C$ is $50 \%$ cumulative. So we can get :

$$
\varepsilon=\sigma \sqrt{-2 \ln (1-0.5)}=1.1774 \sigma \approx 1.18 \sigma
$$

So probable error is 1.8 times of standard variance. Usually $\varepsilon$ is associated with navigational fix error and can be obtained by navigation device query literature [4] schedule 14-1 to schedule 14-3.According to the above conditions, we can get the second constraint conditions:

$$
R \geq \varepsilon
$$

The total probable error of position, $E$,(or the total probable error for short) is the comprehensive standard to measure uncertainty of search target's position (benchmark calculation error) and the work units' ability for accurate navigation, which is also decisive factor to determine specific search region. The plane glide distance $E^{\prime}$ can be worked out under the help of the glide model.

We can figure out $E$ :

$$
E=\sqrt{X^{2}+Y^{2}+D_{\varepsilon}^{2}}
$$

Here we ignore the drift error $D_{\varepsilon}$.So $E$ can be simplified as:

$$
E=\sqrt{X^{2}+Y^{2}}
$$

The error of the search targets $X$ is:

$$
X=F_{i x}+D R_{e}
$$

Here $F i x_{\varepsilon}$ is the search target's positioning error and $D R_{\varepsilon}$ is the error of its estimated path. Fix value can refer to Table 2. $D R_{\varepsilon}$ is proportional to the voyage. Take crashed aircraft's dead reckoning $D R_{\varepsilon}$ as $2 \%$ of the estimated voyage.

The error of the work units is $Y$ :

$$
\begin{gathered}
Y_{i}=F_{i x_{e}}+D R_{e} \\
Y=\sum Y_{i}
\end{gathered}
$$

The $F i x_{\varepsilon}$ is the work units' positioning error and $D R_{\varepsilon}$ is the error of its estimated path. Fix $\varepsilon_{\varepsilon}$ value can refer to Table 2. $D R_{\varepsilon}$ is proportional to the voyage. Take crashed aircraft's dead reckoning $D R_{\varepsilon}$ as $2 \%$ of the estimated voyage.

Table 2. Fix $x_{\varepsilon}$ value

\begin{tabular}{lc}
\hline Positioning method & The positioning error $($ Fix \\
\hline GPS
\end{tabular}

When the plane lost power, the plane glide distance $E^{\prime}$ can be worked out under the help of the glide model. 
Integrated afore-mentioned conditions, finally the value range of $R$ width of the optimal search is:

$$
\left\{\begin{array}{l}
R \leq k r \\
R \geq \varepsilon \\
R \geq E+E^{\prime}
\end{array}\right.
$$

\section{Conclusion}

Now that we have get the baseline and the best search width R, we can work out an optimal search region just as the Figure 2 show.

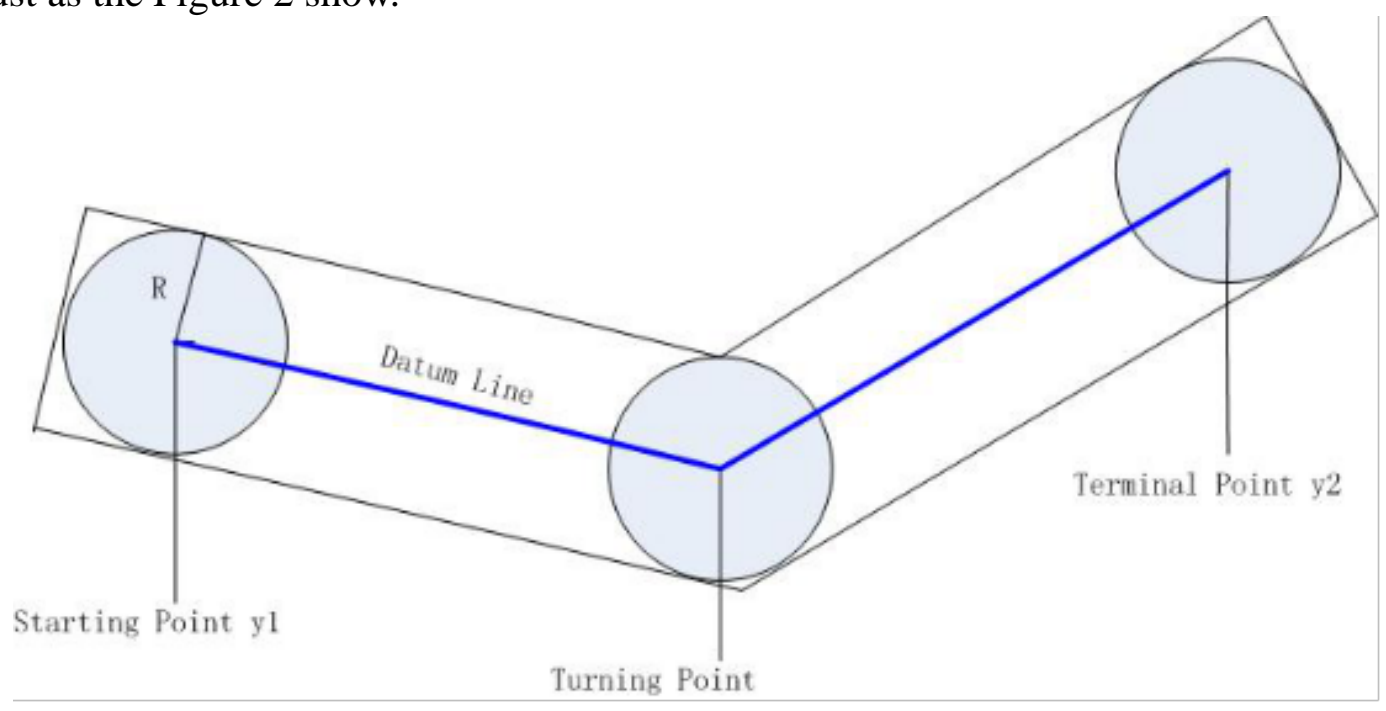

Figure 2. Search region

\section{References}

[1] M. Ceriotti and M. Vasile, “Automated Multigravity Assist Trajectory Planning with a Modified Ant Colony Algorithm,” J. Journal of Aerospace Computing Information and Communication, vol. 7, no. 9, pp. 261-293, 2010.

[2] F. Xhafa, JZ. Sun, A. Barolli, A. Biberaj, and L. Barolli, "Genetic algorithms for satellite scheduling problems,” J. Mobile Information Systems, vol. 8, no. 4, pp. 351-377, 2012.

[3]M. El-Menshawy, J. Bentahar, W. El Kholy, and R. Dssouli, "Verifying conformance of multi-agent commitment-based protocols,” J. Expert Systems With Applications, vol. 40, no. 1, pp. 122-138, 2013.

[4]International aeronautical and maritime search and rescue manual

[5] MA Peng , FENG Jia- hui , HU Chang- yang, "The calculation method on CL /CD of the windmill generator aerofoil ” Huadian Technology, vol.30.no 7,pp. 75-79,2008. 